RESEARCH REPORT

\title{
Prevalence of comorbid psychiatric illness and substance misuse in primary care in England and Wales
}

\author{
Martin Frisher, Juliet Collins, David Millson, Ilana Crome, Peter Croft
}

J Epidemiol Community Health 2004;58:1036-1041. doi: 10.1136/jech.2003.017384

See end of article for authors' affiliations

Correspondence to

Dr M Frisher, Department of Medicines

Management, Keele

University, Staffordshire

ST5 5BG, UK; m.frisher@

keele.ac.uk

Accepted for publication 14 April 2004
Study objective: To estimate the annual period prevalence of co-occurring psychiatric illness and substance misuse among patients in primary care.

Design: Analysis of the general practice research database.

Setting: England and Wales, 1993-1998.

Participants: Registered patients at 230 general practices representing $3.1 \%$ of the population. A comorbid case was defined as one with both a psychiatric diagnosis and substance misuse diagnosis (not including alcohol or tobacco) within a calendar year. A potentially chronic comorbid case was one that met this definition and, in addition, was treated in subsequent years for either a psychiatric condition or substance misuse.

Main results: The annual period prevalence of comorbidity increased from 50/100 000 patient years of exposure (PYE) to 80/100 000 PYE, an increase of $62 \%$ during the study period. Rates of comorbid psychoses, comorbid schizophrenia, and comorbid paranoia increased by $147 \%, 128 \%$, and $144 \%$. The average age of comorbid cases decreased from 38 years to 34 years. Over $80 \%$ of comorbid cases were newly diagnosed in each study year, although many are treated in subsequent years for either psychiatric illness or substance misuse.

Conclusions: This study provides data on the nature and extent of comorbidity in primary care in England and Wales. As the comorbidity rate is increasing by about $10 \%$ each year, and as comorbid cases are becoming younger, it is probable that the comorbidity rate will have increased beyond the study end point.
S ervice provision for patients with concurrent substance misuse and mental health problems poses particular challenges, not least because the medical model of psychiatric services contrasts sharply with the psychosocial orientation of substance misuse services. ${ }^{1}$ Although it is well known that psychiatric patients have high rates of substance misuse and vice versa, the need to undertake epidemiological assessment of the nature and extent of such comorbidity has been identified as a priority. ${ }^{1}$

On the one hand, it has been reported that too many people are being diagnosed as schizophrenic without due consideration being given to the role of drugs and alcohol. ${ }^{2}$ On the other hand, psychiatric illnesses may go undiagnosed when they are attributed to drug use. ${ }^{3}$ Most epidemiological studies have been undertaken in hospital settings and little is known about the scale of comorbidity in the community. The last large scale study to investigate comorbidity in the UK population was the cross sectional OPCS national psychiatric morbidity survey (NPMS) in 1993/4. ${ }^{4}$ Some $2.2 \%$ of the household population aged 16-64 in the UK were rated as "drug dependent" and $1.4 \%$ had two psychiatric conditions (one of which could be drug dependence). Drug dependent respondents were eight times as likely to have a psychiatric condition (mainly depression and anxiety) compared with the general population. It should be noted that the definition of drug dependence used in the NPMS was very broad as it was derived from self response to a number of questions about respondents' experience of drug use. There are no UK data on comorbidity trends over time.

In this study we estimate the nature and extent of comorbidity of psychiatric illness and substance misuse in 230 general practices in England and Wales from 1993 to 1998. These practices represent $3.1 \%$ of the population.

\section{METHODS}

Sample

The sampling frame for this study is the general practice research database (GPRD). The GPRD is the world's largest computerised database of patient records and is owned by the Medicines and Healthcare Products Regulatory Agency (MHRA). Contributing general practitioners record all significant morbidity. GPRD data are subjected to routine quality assessment on the basis of $15 \mathrm{key}$ indicators. The UK government's Office of National Statistics (ONS) supplied the data for this study. The study received ethical approval from the GPRD Scientific and Ethical Advisory Group (SEAG).

Previous research has shown that the classification of psychosis, schizophrenia, affective psychosis, and nonaffective psychosis on the GPRD is accurate, the rate of misclassification is low, and there are few cases not entered onto computer. ${ }^{5}$ GPRD data on drug dependence have been found to be more reliable than other existing surveillance mechanisms. ${ }^{6}$ One important reason is that the GPRD was unaffected by the end of statutory notification of drug dependence in $1997 .{ }^{7}$ We also conducted a validation study in a random sample of patients treated for substance misuse in secondary care settings. Some $92 \%$ of these cases were known to have a diagnosis of substance misuse by their general practitioner.

However, it is known that much substance misuse in the community is not brought to the attention of general

Abbreviations: PYE, patient years of exposure; NPMS, national psychiatric morbidity survey; GPRD, general practice research database; MHRA, Medicines and Healthcare Products Regulatory Agency; ONS, Office of National Statistics; OXMIS, Oxford Medical Information Systems; OPPCS, Office of Population and Census Statistics 
Table 1 One year annual comorbidity prevalence with 95\% confidence intervals, 19931998

\begin{tabular}{llcllc}
\hline Year & $\begin{array}{l}\text { Comorbid cases } \\
\text { within year }\end{array}$ & $\begin{array}{l}\text { Patient years } \\
\text { exposure (PYE) }\end{array}$ & $\begin{array}{l}\text { Rates per } \mathbf{1 0 0 0 0 0} \\
\text { PYE }\end{array}$ & $\begin{array}{l}\text { Lower 95\% } \\
\text { confidence interval }\end{array}$ & $\begin{array}{l}\text { Upper 95\% } \\
\text { confidence interval }\end{array}$ \\
\hline 1993 & 492 & 988735 & 49.76 & 48.38 & 51.17 \\
1994 & 612 & 1002276 & 61.06 & 59.54 & 62.61 \\
1995 & 665 & 1028414 & 64.66 & 64.16 & 65.16 \\
1996 & 702 & 1045543 & 67.14 & 66.98 & 67.30 \\
1997 & 780 & 1056767 & 73.81 & 73.28 & 74.34 \\
1998 & 872 & 1080348 & 80.71 & 80.16 & 81.27 \\
\hline
\end{tabular}

practitioners. There is also evidence that much mental illness goes undetected by the healthcare services. From these findings it follows that the data reported here, which depends on recorded diagnosis by general practitioners, are likely to be minimum estimates of comorbidity in the community as a whole.

\section{Data for this study}

GP practices contributing data continuously between January 1993 and December 1998 were selected for this project. This resulted in data on patients being obtained from 230 practices in England and Wales. The dataset comprised full longitudinal records of all comorbid patients.

\section{Recording of diagnosis on the GPRD}

Diagnoses on the GPRD are recorded using OXMIS codes (Oxford Medical Information Systems) and Read codes. OXMIS codes were devised for use by GPs and are based on the International Classification of Diseases (ICD) and Office of Population and Census Statistics (OPCS) operation codes. Read codes became the standard for diagnostic classification on the GPRD during 1998 and therefore contribute only a small proportion of diagnoses in this study.

There are 1693 OXMIS and Read codes for psychiatric illness. These codes were classified into six diagnostic groups: (a) psychoses, (b) schizophrenia, (c) paranoia, (d) neurosis, (e) personality disorders, and (f) other disorders. The final category covers a wide range of disorders, including "behaviour problems", "hallucinations", "hallucinations auditory", "behaviour antisocial", and "disorder behaviour".

There are 258 OXMIS and Read codes for substance misuse disorders. Whereas psychiatric diagnoses fall into well recognised categories, there is greater latitude when diagnosing substance misuse and furthermore doctors may choose not to code the specific drug involved. A pilot study was undertaken to investigate this issue ${ }^{8}$ and it was decided to

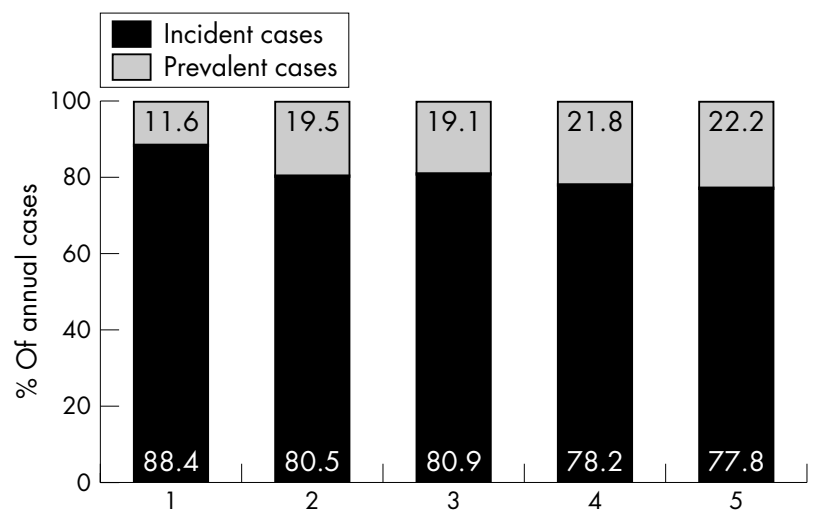

Figure 1 Annual incident and prevalent cases of comorbidity recorded on the GPRD 1994-1998. classify all substance misuse codes into three groups, (a) drug misuse, (b) drug dependence, (c) licit drug dependence.

There were 7025 drug misuse diagnoses recorded in the study period; the main codes used were "drug abuse" (2903), "habitual drug abuse" (2422), "opiate abuse" (788), and "misuse of drugs" (172). Of the 12593 drug dependence codes, the main codes were "drug addiction" (3861), "heroin addiction", (3424), "drug dependence", (3012) and "withdrawal syndrome [drug/narcotic]" (520). Of the 3206 licit drug dependence diagnoses, 3083 (93\%) are accounted for by "benzodiazepine dependence".

This classification does not include alcohol or tobacco related disorders.

\section{Definitions of comorbidity used in this study}

(1) Annual prevalent case. A patient with diagnosed substance misuse and psychiatric illness within a calendar year.

(2) Incident case. From the second study year (1994), an annual prevalent case who had not been comorbid in any of the previous study years.

(3) Continuing prevalent case. From the second study year (1994), an annual prevalent case who had also been comorbid in any of the previous study years.

(4) Potential chronic case. An annual prevalent case who is also seen for either psychiatric illness or substance misuse conditions in subsequent study years.

GPRD denominator years of exposure on the database The nature of general practice registers is such that calculation of disease rates in the general population requires person years of exposure (PYE) for the denominator. PYE are the appropriate unit, rather than numbers of registered cases, because patients' duration of exposure is not fixed. For example, a patient may register on 1 January 1993 and leave the database on 1 January 1994. This patient would have a value of one person year. A case that registered on 1 January 1993 and left on 1 July 1993 would have a value of 0.5 person years representing six months of exposure.

\section{Analysis}

Four numerators were calculated: (a) number of annual prevalent cases (from 1993), (b) number of annual incident cases (from 1994), (c) number of continuing prevalent cases (from 1994), and (d) number of annual cases meeting the definition of potential chronic comorbidity (from 1994). Each numerator was divided by the PYE for that year. This yielded annual measures of (a) annual period prevalence, (b) annual incidence, (c) annual continuing prevalence, and (d) annual prevalence of potential chronic comorbidity.

As the GPRD population for this study is around one million patients per year, the prevalence observed in the study can be expected to closely mirror that in the general population. Confidence intervals were calculated for annual 
Table 2 One year annual comorbidity by age band in 1993 and 1998 and estimated number of cases in England and Wales (E\&W)

\begin{tabular}{|c|c|c|c|c|}
\hline \multirow[b]{2}{*}{ Age group } & \multirow{2}{*}{$\begin{array}{l}1993 \\
\text { Rates per } \\
100000 \text { PYE }\end{array}$} & \multirow{2}{*}{$\begin{array}{l}1998 \\
\text { Rates per } \\
100000 \text { PYE }\end{array}$} & \multirow{2}{*}{$\begin{array}{l}1993 \\
\text { Estimated number of } \\
\text { comorbid cases in E\&W }\end{array}$} & \multirow{2}{*}{$\begin{array}{l}1998 \\
\text { Estimated number of } \\
\text { comorbid cases in E\&W }\end{array}$} \\
\hline & & & & \\
\hline $16-24$ & 88 & 156 & 5078 & 8965 \\
\hline $25-34$ & 82 & 159 & 6874 & 13240 \\
\hline $35-44$ & 64 & 93 & 4609 & 6610 \\
\hline $45-54$ & 46 & 65 & 3142 & 4370 \\
\hline $55-64$ & 38 & 40 & 1923 & 2044 \\
\hline $65-74$ & 26 & 24 & 1163 & 1070 \\
\hline $75-84$ & 30 & 38 & 836 & 1062 \\
\hline $16-84$ & 56 & 93 & 23624 & 37361 \\
\hline
\end{tabular}

period prevalence. ${ }^{9}$ The magnitude of change over the study period was calculated by subtracting the 1993 prevalence figures from the 1998 prevalence figures and expressing the results as percentages.

\section{RESULTS}

\section{Annual period prevalence}

The annual period prevalence of comorbidity increased every year from 1993 to 1998 (see table 1). The estimated number of comorbid cases in the population for England and Wales rose from 24226 in 1993 to 39296 in 1998.

Figure 1 shows that each year the vast majority of annual comorbid cases are incident cases. Although the chances of being a continuing prevalent case increase over time, even in 1998 only $22 \%$ of cases had previously been an annual prevalent case.

\section{Potential chronic comorbidity}

To determine potential chronic comorbidity we examined the records of each cohort of annual comorbid cases. There are two issues that need to be considered. Firstly, are they still registered in subsequent years? Secondly, if they are still registered are they consulting for substance misuse and/or psychiatric illness?

Some $85 \%-88 \%$ of comorbid cases are still registered in the calendar year after their comorbid diagnoses. Five years after diagnoses the figure is still above $50 \%$. Among those cases still registered, $60 \%-66 \%$ of annual comorbid cases had either a substance misuse or a psychiatric diagnosis in the years subsequent to their comorbid diagnoses. In accordance with the definitions given above, the latter group of cases are potential cases of chronic comorbidity.

Figure 2 shows the impact of potential chronic comorbid patients for general practice in England and Wales. Based on these figures, the "average" general practice (total GPRD population divided by the number of GPRD practices) encountered 3.5 potentially chronic comorbid cases in 1994 and 6.8 in 1998. By extrapolation, 11.3 potentially chronic comorbid cases per year are likely to be encountered in the average general practice during 2003.

\section{Annual period prevalence: age band and gender}

Table 2 shows analysis by age band (comorbid patients under 16 and over 84 were excluded from the age band analysis as the numbers were extremely low). There was a significant increase in annual period prevalence of comorbidity in all age groups with the exception of the 65-75 year age band where there was a decline. The average age of comorbid patients decreased from 38 in 1993 to 34 in $1998(F=7.43, \mathrm{p}<0.001)$. From 1993 to 1998, the comorbidity prevalence among men increased by $79 \%$ compared with $44 \%$ for women. In 1993 the male to female gender ratio was 1.1:1 male to female. By 1998, the ratio was 1.37:1.

\section{Annual period prevalence by category of substance misuse}

As explained in the methods section, there are three categories of substance misuse. The annual period prevalence of comorbid drug misuse increased from 18.7/100 000 PYE in 1993 to 36.6/100 000 PYE in 1998 and comorbid drug dependence increased from 26.1/100 000 PYE to 49.6/100 000 PYE.

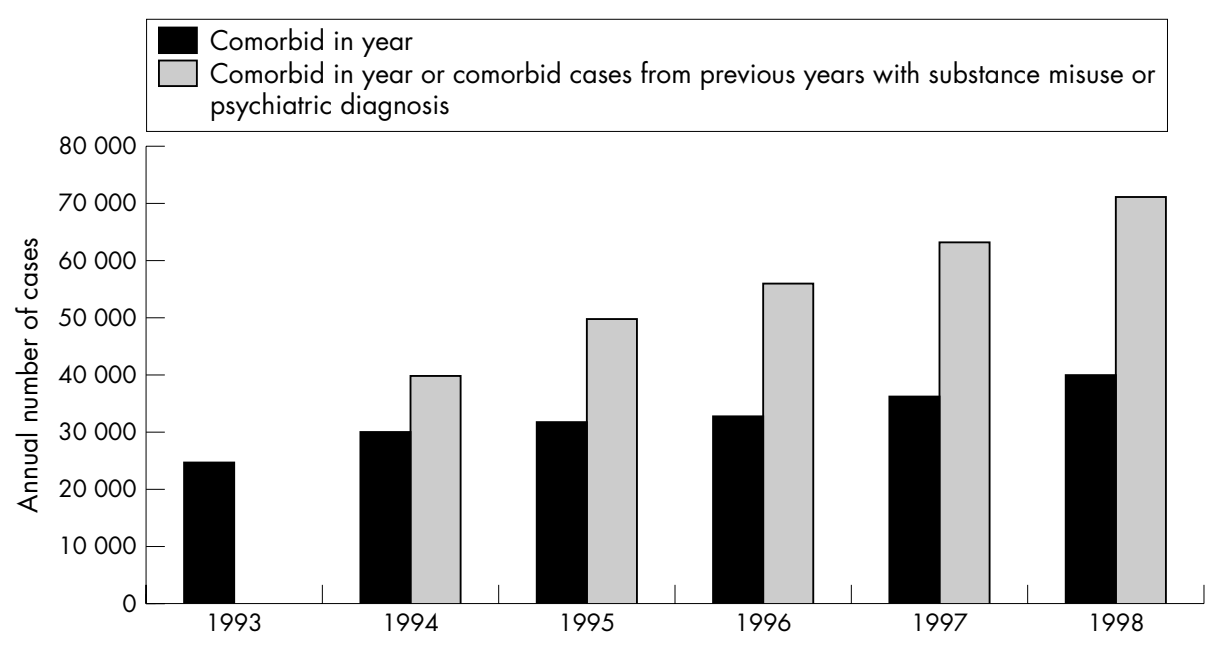

Figure 2 Estimated annual number of comorbid cases and potentially chronic comorbid cases receiving substance misuse or psychiatric treatment in England and Wales. 
Table 3 One year annual prevalence of psychiatric diagnosis for comorbid cases per 100000 patient years exposure on the GPRD

\begin{tabular}{lcccccc}
\hline Year & Psychoses & Schizophrenia & Paranoia & Neurosis & $\begin{array}{l}\text { Personality } \\
\text { disorder }\end{array}$ & Other disorders \\
\hline 1993 & 5.1 & 1.0 & 0.6 & 43.1 & 4.3 & 3.2 \\
1994 & 3.9 & 1.7 & 1.5 & 54.6 & 5.3 & 3.4 \\
1995 & 5.1 & 2.2 & 1.6 & 57.6 & 6.9 & 3.8 \\
1996 & 5.3 & 1.8 & 1.6 & 60.8 & 5.6 & 3.5 \\
1997 & 9.0 & 2.1 & 2.5 & 65.4 & 4.9 & 4.5 \\
1998 & 12.5 & 2.3 & 1.5 & 69.6 & 6.6 & 5.5 \\
\% Rate & 147.1 & 128.8 & 144.1 & 61.6 & 54.7 & 68.7 \\
change 93/ & & & & & & \\
98 & & & & & & \\
\hline
\end{tabular}

Comorbid licit drug dependence rose from 10.6/100 000 PYE in 1993 to $16.1 / 100000$ PYE in 1995 , but there was a noticeable decrease thereafter to 10.8/100 000 PYE in1998.

There were significant differences in the average age of patients at first diagnosis in the three substance misuse categories. The average age of comorbid drug misuse patients, comorbid drug dependent patients, and comorbid licit dependent cases was 30.2, 33.4, and 45.3 years respectively. The overall $F$ ratio was $320.4, \mathrm{p}<0.001$ and post hoc comparisons of the three comorbid substance misuse categories were all significant at $\mathrm{p}<0.001$.

\section{Annual period prevalence by category of psychiatric illness}

The annual period prevalence in all comorbid psychiatric diagnostic groups increased during the study period. The percentage rate of change between 1993 and 1998 (see table 3) ranged from $54.7 \%$ (comorbid personality disorders) to $147.1 \%$ (comorbid psychosis).

The average (mean) age in years at first diagnosis was (in ascending order): personality disorders 31.4, schizophrenia 32.0, paranoia 32.2, other disorders 33.7, neurosis 34.9, and psychoses 39.4. The overall $F$ ratio in comparing ages between these groups was 20.3, $<<0.001$. Post hoc comparisons show

\section{Policy implications}

- The apparently growing comorbidity of psychiatric illness and substance misuse among patients in primary care has important implications for resource allocation, training, and service organisation.

- Further longitudinal exploration of comorbidity in primary care is important as the GPRD is uniquely British and of greater relevance than the large body of research undertaken in the United States. Analysis of the period 1999-2003 will provide further information on the impact of comorbidity on primary care.

- Future work should focus on the relation between physical and psychiatric comorbidity. It should also consider alcohol and smoking as well as substance misuse.

- Given rising comorbidity it is important to determine what assessments and interventions in primary care are needed to provide effective patient care. It is important to improve competencies in primary care teams, taking into account the changing problems they face in the management of substance misuse and psychiatric illness. that the age of patients at first diagnosis of comorbid psychoses was higher than the age of first diagnosis in the other comorbid psychiatric groups.

\section{DISCUSSION}

\section{Strengths and weaknesses of the study}

The strengths of the GPRD are that it is based on registered patients and therefore represents consulting rates in the general population and that data are collected routinely. The GPRD practices record substance users who are unlikely to appear in analyses of problematic drug use (for example, elderly misusers of benzodiazepines), but it will miss people whose mental illness or drug use never results in primary care service contact. Patients who do not remain registered at the same GPRD practice are lost to follow up. However, as has been previously discussed with reference to autism ${ }^{10}$ and drug dependence, ${ }^{6}$ GPRD trend data are not susceptible to many of the biases in, for example, population surveys or case registers. The database contains little information on specific drug of misuse or dependence because, as explained in the methods section, most doctors only code substance misuse at the most general level available in the Read or OXMIS hierarchy.

\section{Prevalence of comorbidity}

The observed increase in annual period prevalence represents an estimated increase in the annual number of comorbid patients seen in general practice in England and Wales from 24226 to 39296 between 1993 to 1998. If the rate of increase observed from 1993-1998 has been maintained the extrapolated number of patients will be 53268 in 2003. Based on the definition of potential chronic comorbidity, there are an estimated 117389 patients being seen in general practice in 2003. Although treatment of comorbidity is frequently discussed in the context of specialist services, ${ }^{4}$ our results show that general practice is playing a growing part in the management of the range of comorbid substance misuse/ psychiatric conditions.

These figures relate to our study definition of comorbidity, which need not be the simultaneous occurrence of both substance misuse and a psychiatric condition. However, a one year period is a reasonable length of time because in practice it is often very difficult to be certain of a comorbid diagnosis in a patient who is misusing substances. There are many reasons why the two diagnoses may not be simultaneous. Substance misuse might have a set of symptoms directly related to the pharmacological effect or to withdrawal, or which may overlap with a psychiatric diagnosis. It may only be possible to diagnose the "co-occurring" but separate condition once the patient is drug free. Alternatively, if the practitioner knows the pattern of presenting symptoms and illness in a person over some time and can recognise when 
the substance misuse is "destabilised" by, for example, depression, anxiety, or a psychotic illness. Thus, while the clinical conditions may be occurring simultaneously, it requires an understanding of the temporal sequencing of the symptoms for the GP to make the diagnoses of substance misuse and mental illness.

\section{Comorbidity according to type of psychiatric illness}

Previous reviews have focused on the co-occurrence of psychoses and substance misuse ${ }^{1}$ and in this study the prevalence of comorbidity related to psychoses increased by 147\% between 1993 and 1998. While this rate of increase was higher than for any other type of comorbid psychiatric condition, comorbidity in relation to neuroses (depression and anxiety) are the most frequent psychiatric conditions recorded by general practitioners.

National general practice data published by $\mathrm{ONS}^{11}$ indicate that the prevalence of schizophrenia in England and Wales increased by $6 \%$ from 1994 to 1998. In contrast, the prevalence of comorbidity related to schizophrenia in our study increased by $128 \%$. Other severe forms of comorbid mental illness in our study also rose substantially; paranoia by $144 \%$ and psychoses by $147 \%$.

\section{Comorbidity according to type of substance misuse}

The increases in one year period prevalence of comorbid drug misuse and drug dependence over the six year study period were $95 \%$ and $85 \%$ respectively. This compares with an increase of $70 \%$ in all forms of treated substance abuse on the Regional Drug Misuse Databases from 1993 to $1998 .^{12}$ Comorbid licit drug dependence in this study only rose by $2 \%$. This figure conceals two patterns. From 1993 to 1995 the prevalence of comorbid licit drug dependence increased by $52 \%$, while from 1995 to 1998 the rate fell by $49 \%$. These figures may reflect concern about benzodiazepine prescribing. In 1994, the Department of Health issued guidelines on the prevention of benzodiazepine dependence. Another reason may have been the withdrawal of temazepam capsules after concern about their misuse by drug injectors. ${ }^{13}$ As we have noted elsewhere, ${ }^{6}$ the GPRD seems to provide reliable data on trends in treated problematic drug use, with the caveat that many doctors do not record the specific drugs.

The types of psychiatric illness seen among comorbid drug misuse and drug dependence patients were fairly similar. For example $17 \%$ of diagnoses among comorbid drug misuse patients were for schizophrenia or psychoses compared with $19 \%$ among comorbid drug dependent patients. This suggests that serious comorbid mental illnesses such as psychoses and schizophrenia are almost as prevalent in different phases of drug users' careers, although further work is required to investigate this hypothesis.

\section{Comorbidity by categories of age and gender}

Unlike many studies of comorbidity that tend to focus on young adults, this study covers the adult age spectrum (16+). Although the highest prevalence rates were observed among patients aged 25-34 (159/100 000), this was closely followed by the rate among those aged 16-24 (156/100 000). In terms of the psychiatric classification, the "other disorders" have the youngest average age ( 25.2 years). This reflects diagnoses such as "antisocial behaviour" and "behaviour problem". However, only $4 \%$ of diagnoses in the 16-24 age category were recorded in the "other diagnoses" category. At the other end of the spectrum, "psychoses" had the highest average age (37.6 years) probably because this category includes "confusion" and "dementia". The average age of comorbid patients during the study period fell from 38 to 34 years. Although the average age has fallen, it is still higher than the average of clients reported to the Regional Drug Misuse
Databases in 2001, which was 28.4 years. At the start of the study in 1993 the male:female ratio was about 1:1 but by 1998 the ratio was 1.4:1. This may reflect the increasing primacy of substance misuse in comorbidity, although further analysis will be required to evaluate this hypothesis.

\section{Conclusions}

The results of this study show that GPs are seeing more comorbid patients each year. The average general practice saw seven potentially chronic comorbid patients in 1998 and on current trends this will increase to 14 in 2006. Furthermore, comorbidity rates are rising more rapidly for serious psychiatric conditions.

Comorbidity, as defined in this study, covers a wide range of substances, psychiatric conditions, and causal mechanisms. ${ }^{14}$ This complexity makes it difficult to assess service provision or effectiveness, and indeed much of the discussion has been confined to clinical populations in contact with psychiatric services. ${ }^{4}$ A review of services for drug misusers in 1996 recommended that all drug misusers need to have access to primary care through normal registration with a GP and that GPs are well placed to identify and offer advice to drug misusers who may not be in touch with specialist agencies. ${ }^{15}$ The role of the general practitioner in providing shared care for substance misusers is increasingly being encouraged ${ }^{16}$ and family doctors are increasingly becoming the first point of contact for young drug misusers, ${ }^{17}$ although misgivings have been expressed about treatment of drug users in primary care. While this study is not an evaluation of these policies, it provides epidemiological information to inform the policy debate on issues related to the management of comorbidity in the UK.

\section{ACKNOWLEDGEMENTS}

We are extremely grateful to the following people: Velda Osborne at the Office of National Statistics who has helped clarify the study design, supplied us with the data for the study, and answered endless questions with infinite patience; Gazala Akram who played a key part in developing the study and provided comments on the manuscript. The authors would like to thank the General Practice Research Database team at the Medicines and Healthcare Products Regulatory Agency, the Office of National Statistics and contributing general practitioners and their patients. The views expressed in the paper are those of the authors and not the Department of Health who funded the study.

\section{Authors' affiliations}

M Frisher, J Collins, Department of Medicines Management, Keele University, Staffordshire, UK

D Millson, General Practitioner, Leek, UK

I Crome, Academic Psychiatry Unit, Keele University Medical School

P Croft, Primary Care Sciences Research Centre, Keele University

Funding: the study was enabled by a research grant from the Department of Health under the Drug Misuse Research Initiative.

Conflicts of interest: none declared.

\section{REFERENCES}

1 Weaver T, Renton A, Stimson G, et al. Severe mental illness and substance abuse. BMJ 1999;318:137-8.

2 Cohen SI. Overdiagnosis of schizophrenia: role of alcohol and drug misuse. Lancet 1995;346:1541-2.

3 Anonymous. Judge says doctors failed knifeman who saw Beatles on broomsticks. Daily Telegraph 2000;16 Nov.

4 Farrell M, Howes S, Taylor C, et al. Substance misuse and psychiatric comorbidity: an overview of the OPCS national psychiatric morbidity survey. Addict Behav 1998;23:909-18.

5 Nazareth I, King M, Haines A, et al. Accuracy of diagnosis of psychosis on general practice computer system. BMJ 1993;307:32-4

6 Frischer M, Norwood J, Heatlie $\mathrm{H}$, et al. A comparison of trends in problematic drug misuse from two reporting systems. J Public Health Med 2000;22:362-7.

7 Tregoning D. Home Office addicts index no longer exists. BMJ 1998;316:151. 
8 Frischer M, Akram G. Prevalence of comorbid mental illness and drug use recorded in general practice: preliminary findings from the General Practice Research Database. Drugs: Education, Prevention and Policy 2001;8:276-80.

9 Fliess J. Statistical methods for rates and proportions. New York: Wiley, 1981.

10 Kaye J, Melero-Montes $M$, Jick H. Mumps, measles, and rubella vaccine and the incidence of autism recorded by general practitioners: a time trend analysis. BMJ 2001;322:460-3.

11 Office of National Statistics. Key statistics in general practice 1998. London: National Statistics, 2000.

12 National Statistics. Statistics from the regional drug misuse databases for the six months ending September 2000. London: National Statistics, 2001.

13 Dyer C. Company appeals to retain temazepam capsules. BMJ 1996;312:659-60.
14 Crome IB. Psychiatric co-morbidity and substance misuse: towards improved service provision. Drugs: Education, Prevention and Policy 1999;6:151-74.

15 Department of Health. Task force to review services for drug misusers: report of an independent review of drug treatment services in England. London: Department of Health, 1996.

16 Gerada C, Tighe J. A review of shared care protocols for the treatment of problem drug use in England, Scotland and Wales. Br J Gen Pract 1999;439:125-6.

17 Department of Health Scottish Office, Department of Health Welsh Office, Department of Health and Social Services, Northern Ireland. Drug misuse and dependence-guidelines on clinical management drug misuse and dependence-guidelines on clinical management. London: Stationery Office, 1999.

\section{$\mathrm{ECHO}$}

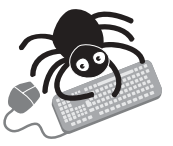

Please visit the Journal of Epidemiology and

Community Health website [www.jech. com] for a link to the full text of this article.
An investigation into general practitioners associated with high patient mortality flagged up through the Shipman inquiry: retrospective analysis of routine data Mohammed A Mohammed, Anthony Rathbone, Paulette Myers, Divya Patel, Helen Onions, Andrew Stevens

Objective: To identify a credible explanation for the excessively high mortality associated with general practitioners who were flagged up by the Shipman inquiry.

Design: Retrospective analysis of routine data.

Setting: Primary care.

Participants: Two general practitioners in the West Midlands who were associated with an unacceptably high mortality of patients during 1993-2000.

Main outcome measures: Observed and expected number of deaths and deaths in nursing homes.

Results: Preliminary discussions with the general practitioners highlighted deaths in nursing homes as a possible explanatory factor. No relation was found between the expected number of deaths and deaths in nursing homes in each year during 1993-2000 for either general practitioner. In contrast, the magnitude and shape of the curves of a cumulative sum plot for excess number of deaths (observed minus expected) in each year were closely mirrored by the magnitude and shape of the curves of the number of patients dying in nursing homes; and this was reflected in the high correlations $\left(\mathrm{R}^{2}=0.87\right.$ and 0.89$)$ between excess mortality and the number of deaths in nursing homes in each year for the general practitioners. These findings were supported by administrative data.

Conclusions: The excessively high mortality associated with two general practitioners was credibly explained by a nursing home effect. General practitioners associated with high patient mortality, albeit after sophisticated statistical analysis, should not be labelled as having poor performance but instead should be considered as a signal meriting scientific investigation.

\ $B M J$ 2004;328:1474-1477. 